Article

\title{
Partial Pre-Emphasis for Pluggable 400 G Short-Reach Coherent Systems
}

\author{
Ahmad Abdo ${ }^{1, *}$, Xueyang Li ${ }^{2}$, Md Samiul Alam ${ }^{2}$, Mahdi Parvizi ${ }^{3}$, Naim Ben-Hamida ${ }^{3}$, \\ Claude $D^{\prime}$ Amours ${ }^{1}$ and David Plant ${ }^{2}$ \\ 1 School of Electrical Engineering and Computer Science, University of Ottawa, Ottawa, ON K1N 6N5, \\ Canada; cdamours@uottawa.ca \\ 2 Department of Electrical and Computer Engineering, McGill University, Montreal, QC H3A 2A7, Canada; \\ xueyang.li@mail.mcgill.ca (X.L.); md.samiul.alam@mail.mcgill.ca (M.S.A.); david.plant@mcgill.ca (D.P.) \\ 3 Ciena Corporation, Ottawa, ON K2K 0L1, Canada; mparvizi@ciena.com (M.P.); \\ nbenhami@ciena.com (N.B.-H.) \\ * Correspondence: aabdo013@uottawa.ca
}

Received: 2 November 2019; Accepted: 10 December 2019; Published: 11 December 2019

\begin{abstract}
Pre-emphasis filters are used to pre-compensate for the transmitter frequency response of coherent systems to mitigate receiver noise enhancement. This is particularly essential for low-cost, low-power coherent transceivers due to having an extremely bandlimited transmitter. However, the pre-emphasis filter also increases the signal peak-to-average power ratio (PAPR), thus posing a higher effective number of bits $(\mathrm{ENoB})$ requirement for the arbitrary waveform generator (AWG). In this paper, we first numerically study the PAPR impact of partial pre-emphasis filters. We show that with partial pre-emphasis, an ENoB reduction from 5 to 4.5 bits is attainable at the same signal-to-noise ratio (SNR) out of the AWG. Next, we experimentally investigate the overall performance penalty of partial pre-emphasis in a 50 Gbaud 16QAM coherent system. A manageable $\mathrm{Q}$ factor penalty of around $0.5 \mathrm{~dB}$ is found for both single-polarization and dual-polarization systems with a $0.8 \mathrm{~dB}$ PAPR reduction.
\end{abstract}

Keywords: optical communications; pluggable transceivers; pre-emphasis; coherent communications

\section{Introduction}

The high-capacity, high-spectral efficiency and low operating expenses (OPEX) of coherent systems have made them attractive candidates for $400 \mathrm{G}$ data center interconnects (DCI) as standardized in 400ZR by optical internetworking forum (OIF) [1]. Due to costly rack space in data centers, it is imperative to implement integrated coherent transceivers to fit small-sized pluggables such as Quad Small Form Pluggable-Double Density (QSFP-DD) to provide high switch-port density. This can be achieved using either silicon or InP-based technologies. The silicon photonics ( $\mathrm{SiPh}$ ) platform has an edge over its InP counterpart in terms of cost-effectiveness, since it leverages the complementary metal-oxide semiconductor (CMOS)-compatible process in silicon foundries.

Silicon photonic traveling wave modulators (TWMZMs) have been actively studied and show good potential for coherent applications [2-9]. To meet the low power requirements in $400 \mathrm{G} \mathrm{SiPh}$ coherent systems, short TWMZMs with low $\mathrm{V}_{\pi}$ are attractive, which allow for low-power radio frequency (RF) driver amplifiers (DA). However, this is obtained by trading off the modulator E-O bandwidth, which leads to the transmitter (Tx) low-pass filtering that is significantly stronger than the receiver (Rx) [8]. Furthermore, this is aggravated by the cascaded low-pass frequency response of the arbitrary waveform generator (AWG), which often possesses a comparably low bandwidth in the entire channel. 
It is known that linear equalizers at the receiver can compensate for the frequency response of the entire channel, including the severely band limited Tx, but also incur undesired noise enhancement. One way to deal with the low bandwidth of the system is to use partial response signaling like duobinary (DB) modulation format or Tomlinson-Harashima precoding (THP) [10-13]. However, both these solutions require an increased number of signal levels such that higher signal-to-noise ratio (SNR) is required for the same forward error correction (FEC) threshold. Moreover, DB FFE has either modulo loss due to modulo operation or increased DSP complexity due to the use of MLSE. Trellis coded modulation (TCM), though promising, increases transmitter and receiver complexity significantly [13]. So, a finite-impulse response (FIR) pre-emphasis filter is usually used at the Tx side to pre-compensate the Tx frequency response. Several works have been conducted on transmitter pre-emphasis targeting high-speed coherent systems [14-17]. Authors in [14,15] proposed a pre-emphasis filter for the AWG frequency response, and in [16] the authors presented an adaptive digital pre-distortion (DPD) algorithm to mitigate the undesirable linear and non-linear distortions of the AWG, digital to analog converter (DAC), and dual-polarization Mach-Zehnder modulator (DP-MZM). However, pre-compensating for the entire Tx low-pass filtering leads to increased signal peak-to-average ratio (PAPR) and reduced average signal power out of the AWG, thus degrading the transmitter signal-to-noise-ratio (SNR). We demonstrate in this work that by using a partial pre-emphasis filter, a lower signal PAPR is achieved and a higher signal power is obtained from the AWG. The work is part of the quest to understand and, ultimately, develop low-power transceivers that do not depend on the modulator driver, i.e., radio frequency amplifications.

In this paper, we first numerically study the change of the signal PAPR when the pre-compensation of the transmitter is reduced to varying degrees for a 50 Gbaud 16QAM signal. It is found that the effective number of bits (ENoB) requirement can be reduced by around 0.5 by using a partial pre-emphasis filter for the same transmitter signal SNR. Next, using discrete optical equipment, we experimentally characterize the overall performance penalty of partial pre-emphasis for a 50 Gbaud 16QAM short-reach coherent system with extremely bandlimited Tx. A $0.4 \mathrm{dBQ}$ factor penalty is observed in single-pol case for a partial pre-emphasis filter with a frequency response drop of $6 \mathrm{~dB}$ at $20 \mathrm{GHz}$ and for a dual-pol coherent system with polarization-dependent loss (PDL) and polarization-mode dispersion (PMD); this penalty is found to be $0.5 \mathrm{~dB}$. We conclude that the use of pre-emphasis filter can relieve some ENoB requirement of the AWG at the cost of a manageable performance penalty.

The paper is organized as follows: an introduction to the SiPh modulators and model used is highlighted in Section 2. In Section 3, we present a numerical study of the PAPR change versus the extent of Tx pre-compensation. The experimental results and associated discussions are presented in Section 4. Design trade-offs and engineering advantages of partial pre-emphasis are discussed in Section 5 .

\section{Silicon Photonics Modulators}

$\mathrm{SiPh}$ modulators are used at the front-end of the optical transponder to modulate a light beam produced by a tunable laser. The authors of this paper, in [2], investigated push-pull modulator SiPh MZM fabricated in an Silicon-on-Insulator (SOI) process with low resistivity. As discussed by the authors in [9], the length of the modulator transmission medium and the applied voltage bias determine the resulting frequency response $\left(\mathrm{S}_{21}\right)$. It was shown that the shorter the modulator, the higher is the $3 \mathrm{~dB}$ bandwidth when measured using small signal electro-optics (EO) measurements. The resulting frequency response with a bias voltage of $4 \mathrm{~V}$ is shown in Figure 1. When modulator' RF drivers are used to boost the low voltage coming from CMOS digital to analog converter (DAC), a balanced RMS voltage is required. If the value is too high, it risks operating in the non-linear region of modulator (edges of the S-curve) and higher power consumption; if too low, it risks more carrier leak, which causes constellation to shift and degrades SNR. 


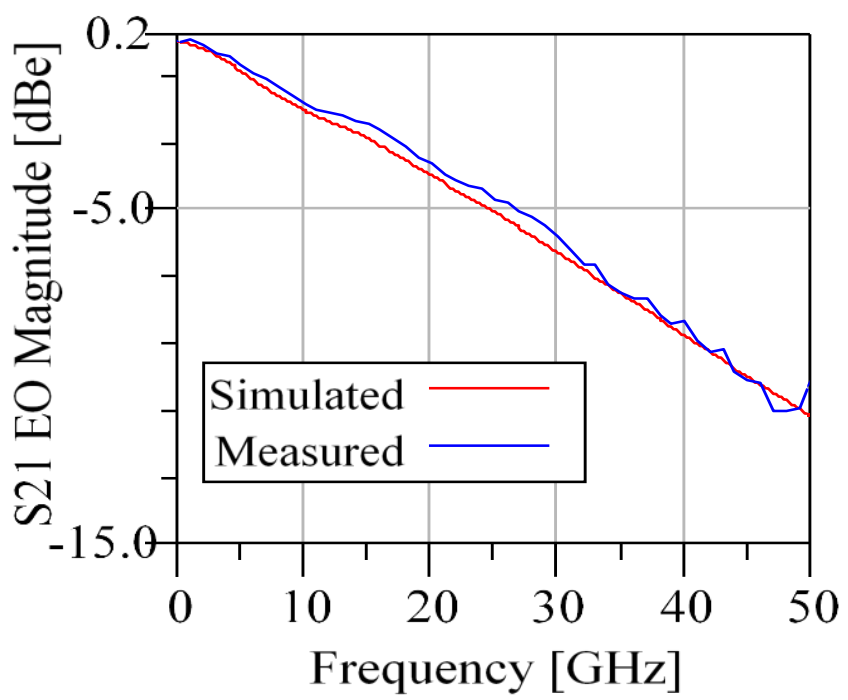

Figure 1. Simulated and measured electro-optics (EO) response at $4 \mathrm{~V}$ reverse bias for a $4 \mathrm{~mm}$ MZM [9].

\section{Pre-Emphasis in Layer-0 Optical Systems}

Pre-emphasis filter is commonly used in optical communication systems to pre-compensate for part of or the entire channel inter-symbol interference (ISI), such that the noise enhancement associated with Rx equalization is alleviated. Nonetheless, the pre-emphasis filter applied to the signal at the Tx also burdens the AWG used to generate the RF signal due to increased signal PAPR. This leads to lower signal root-mean-square (RMS), and equivalently lower signal power out from the AWG, thus degrading the signal SNR when the power of the quantization noise, the thermal noise, as well as the harmonic noise are relatively stable. In this section, we present a simulation model to investigate the impact of the pre-emphasis filter on the signal SNR out from the AWG.

Figure 2 shows the simulation setup. The digital signal, $S(\Omega)$, is generated and filtered by a root raised cosine (RRC) pulse shaper, $\sqrt{N(\Omega)}$, with a roll-off factor of 0.125 , followed by a digital pre-emphasis (DPE) filter. The AWG frequency response is modelled as a 3rd order Bessel filter with $14 \mathrm{GHz}$ of $3 \mathrm{~dB}$ BW operating at $64 \mathrm{GSa} / \mathrm{s}$. If we ignore clipping, the quantization noise, $n_{q}(\Omega)$, can be approximated as white noise, and the noise variance is given as $\Delta^{2} / 12$, with $\Delta$ being the quantization step size. If the input to the DAC after pre-emphasis filter is $X(\Omega)$, we can derive

$$
\Delta=\frac{x_{\max }}{2^{\text {ENOB-1 }-1}}=\frac{\sqrt{\sigma_{x}^{2} \cdot 10^{P A P R / 10}}}{2^{\text {ENOB-1 }-1}}
$$

where PAPR is in $\mathrm{dB}$ and $\sigma_{x}^{2}$ is variance of signal fed into the DAC, $X(\Omega)$. So, this quantization step size, $\Delta$, depends on signal PAPR and ENoB. For the purpose of simplifying the comparison between different cases, we consider the nominal ENoB. An additional AWGN noise, $\mathrm{n}_{\mathrm{add}}(\Omega)$, is added along with the quantization noise to better match the practical AWG [18]. This noise is related to the SNR within the AWG module, and, as shown in reference [18], this model shows considerable match with measurements and numerical simulation. The DPE is calculated using least mean square (LMS) algorithm considering only the limited bandwidth of the AWG at the beginning and then used for all other results. 


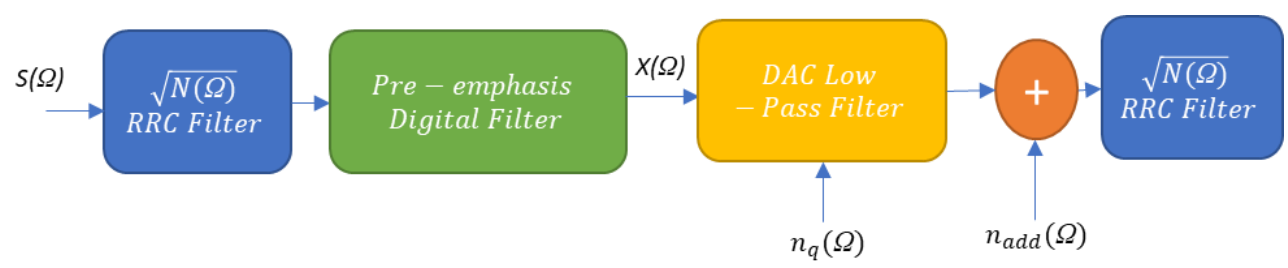

Figure 2. Simulation setup.

Figure 3 shows the frequency response of the complete and partial-pre-emphasis filters created based on the full pre-emphasis filter. Here, by "complete pre-emphasis" we mean LMS-based pre-emphasis filter that pre-compensates for the frequency response of the transmitter, and thus the transmitted spectrum right after the AWG is equal to set pulse shaping. For partial pre-emphasis, we multiply this complete pre-emphasis filter via an attenuation function. This attenuation function is a linear function with a certain amount of attenuation at a fixed frequency. The partial-pre-emphasis filter shown in Figure 3 has a $3 \mathrm{~dB}$ reduction of frequency response at $20 \mathrm{GHz}$. In the rest of the simulation, the partial pre-emphasis filters are obtained by reducing the frequency response at $20 \mathrm{GHz}$ to different extent. As we stated earlier by doing the complete pre-emphasis, we increase the signal PAPR degrading the signal SNR at the output of the modulator. Figure 4 plots the PAPR of transmitting signal into the AWG as a function of the magnitude loss at $20 \mathrm{GHz}$. It is seen that the PAPR decreases almost linearly with the frequency loss and there is $1.3 \mathrm{~dB}$ reduction of the PAPR for a $4 \mathrm{~dB}$ loss at $20 \mathrm{GHz}$.

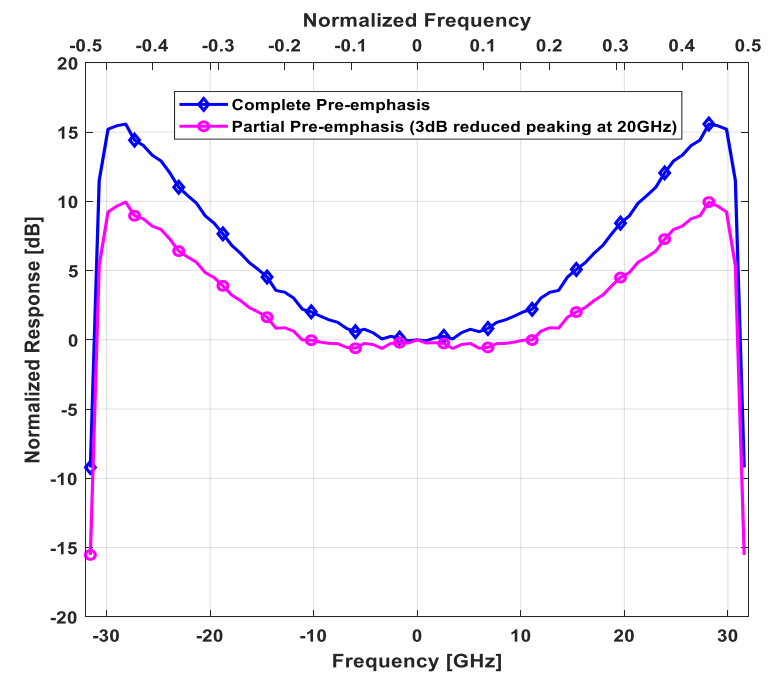

Figure 3. Frequency response of the digital pre-emphasis (DPE) filter.

This reduction in PAPR increases the signal SNR. To calculate the transmitted signal SNR, we use a matched filter followed by an 11-tap feed-forward equalizer (FFE) at the receiver. The FFE removes the ISI caused by partial pre-emphasis. Figure 5 plots the change of the signal SNR out of the AWG versus different losses at $20 \mathrm{GHz}$ when the ENoB varies from 4 to 6 bits. It can be observed that the SNR increases with increasing loss for different ENoB values. This is a consequence of boosting the average signal power relative to the noise-plus-distortion power when the signal PAPR is reduced with higher loss, as shown in Figure 4. The increase is more pronounced for lower ENoB values. With $4 \mathrm{~dB}$ reduced peaking with ENoB of 4.5 bits, we can get similar signal SNR as resulted from using an ENoB value of 5 and complete pre-emphasis. This indicates that by applying partial pre-emphasis filters, the ENoB requirement of the AWG can be relieved such that lower cost coherent Tx can be built. 


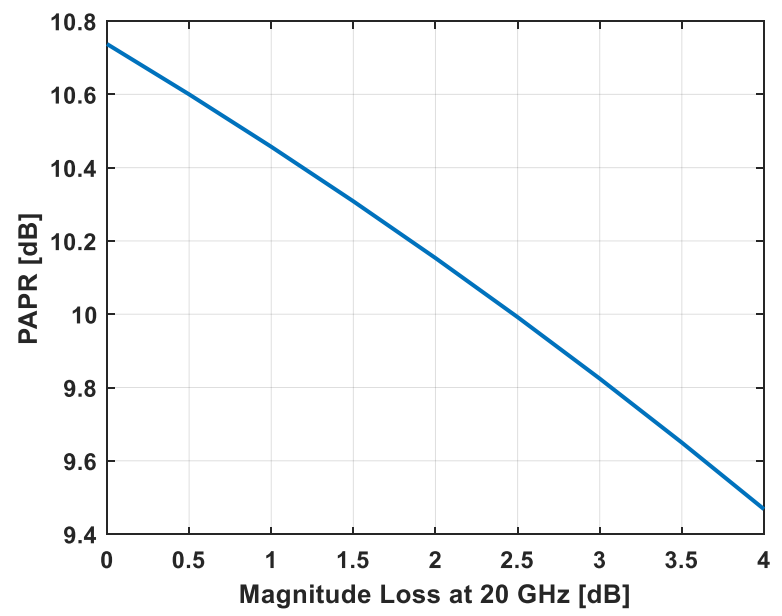

Figure 4. PAPR vs. Tx loss at $20 \mathrm{GHz}$.

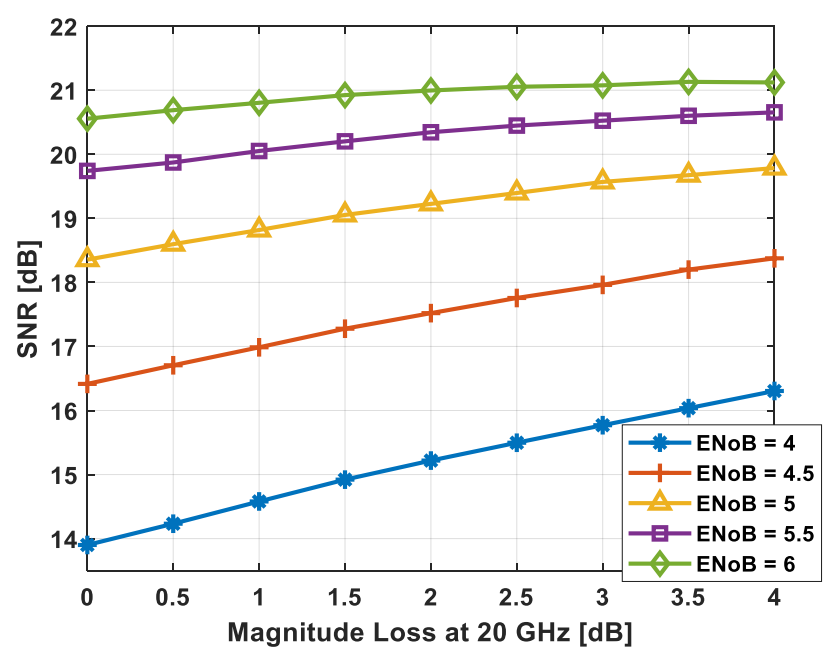

Figure 5. Signal signal-to-noise ratio (SNR) vs. Tx loss at $20 \mathrm{GHz}$.

\section{Experimental Setup and DSP}

Figure 6 shows the experimental setup used to evaluate the impact of partial pre-emphasis. First, 16QAM symbols are generated in the Tx DSP and then resampled to the AWG sampling rate at $64 \mathrm{GSa} / \mathrm{s}$ for pulse shaping via the root-raised-cosine (RRC) matched filter. Next, a pre-emphasis filter is applied to the signal to pre-compensate for the frequency response of the transmitter. The filter taps are obtained from a T/2-spaced feedforward equalizer that compensates for the whole transmitter frequency response including modulator. Note that in the experiment, we reduce the peaking of the pre-emphasis filter to different levels to achieve partial pre-emphasis of the Tx. Then, the signal is quantized and fed to the AWG memory for digital-to-analog conversion. The output RF signals from the two-channel AWG are amplified by a pair of RF amplifiers with a $3 \mathrm{~dB}$ bandwidth of $42 \mathrm{GHz}$ and then modulated onto the optical carrier at $1550.12 \mathrm{~nm}$ through an In-phase/Quadrature modulator (IQM). In the setup, a $\mathrm{LiNbO}_{3} \mathrm{IQM}$ with a $3 \mathrm{~dB} \mathrm{E}-\mathrm{O}$ bandwidth of $15 \mathrm{GHz}$ is used to emulate a low bandwidth SiPh modulator. The power of the optical source was $15.5 \mathrm{dBm}$ and LO power was set to $12 \mathrm{dBm}$. The launch power into the fiber was around $-9 \mathrm{dBm}$ for $50 \mathrm{Gbaud}$ signal. After transmission over $80 \mathrm{~km}$ of standard single-mode fiber (SSMF), the optical signal is pre-amplified by erbium-doped fiber amplifier (EDFA) with a noise figure of $5 \mathrm{~dB}$ and received by the coherent receiver (CRX). The CRX consists of an optical hybrid followed by two balanced photodetectors (BPD) with no inline transimpedance amplifier (TIA). The balanced photodetectors have a $3 \mathrm{~dB}$ bandwidth 
of $45 \mathrm{GHz}$. An 8-bit real-time oscilloscope (RTO) operating at $160 \mathrm{GSps}$ and $62 \mathrm{GHz}$ bandwidth is used to capture the data for subsequent offline signal processing. An ASE source and variable optical attenuator (VOA) is used at the receiver to control the received signal optical signal-to-noise ratio (OSNR). The sampled signal from RTO is stored and processed following common coherent DSP algorithms, which includes resampling, frequency offset compensation, dispersion compensation, RRC matched filtering, and phase locked-loop (PLL)-interleaved equalization to compensate for both the ISI and the phase noise. Finally, the symbols are determined and decoded for bit-error rate (BER) calculation.

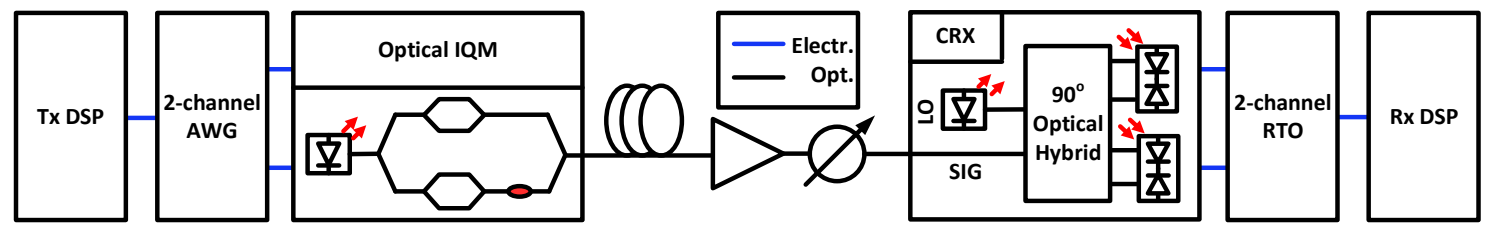

Figure 6. Experimental setup.

\subsection{Results and Discussion}

Although by reasonably reducing the peaking of the partial pre-emphasis filter, the SNR of the signal from the AWG is increased as discussed before, the reliance on the Rx equalizer to compensate for the remainder of the Tx frequency response and the Rx frequency response results in increased noise enhancement, especially for low-cost coherent systems with extremely bandlimited Tx. This could result in overall performance penalty that is experimentally characterized in this section.

\subsubsection{Impact of Partial Pre-Compensation on Q-Factor in a Single-Polarization Coherent System}

We first investigate the performance penalty of partial pre-compensation relative to full pre-compensation in a 50 Gbaud 16QAM single-pol coherent system in back-to-back. Note that we use a low bandwidth $\operatorname{Tx}(10 \mathrm{GHz})$ in the experiment to better study the impact of partial pre-compensation on the performance of a low-cost, low-power coherent system. Though this limits the system from working at 60 Gbaud for 400ZR, the conclusion is still valid. The black $\left(^{*}\right)$ and red $(+)$ curves in Figure 7 show the Q-factor penalty of partial pre-compensation versus the reduced frequency peaking of the pre-emphasis filter at $20 \mathrm{GHz}$ obtained from experimental and numerical results, respectively.

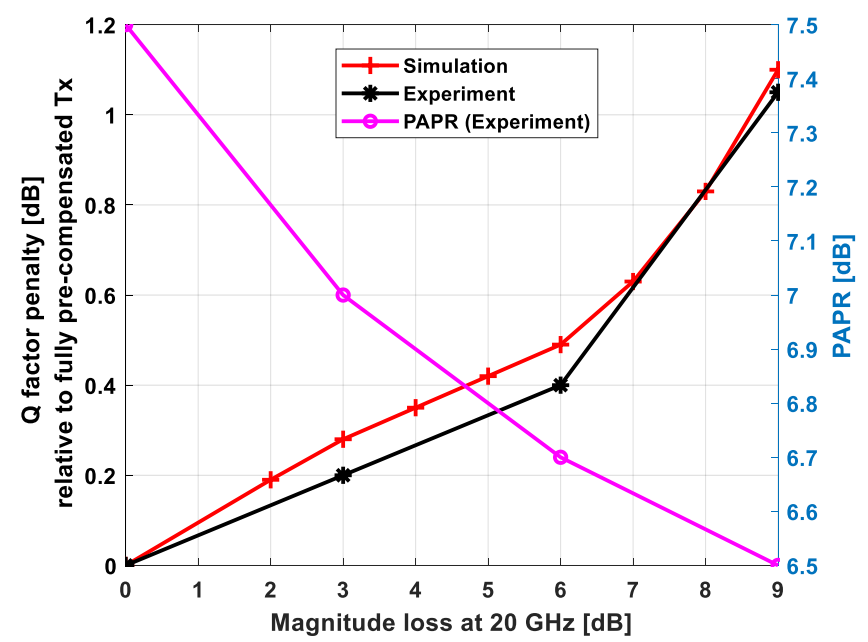

Figure 7. Q-Factor penalty versus loss at high-frequency.

It is consistently found for the two curves that the $Q$ factor penalty increases with increasing attenuation at $20 \mathrm{GHz}$. The black $\left(^{*}\right)$ curve indicates in the experiment that a $0.4 \mathrm{~dB} \mathrm{Q}$ factor penalty 
occurs at $6 \mathrm{~dB}$ attenuation at $20 \mathrm{GHz}$. This is due to increased noise enhancement at the receiver, since more $\mathrm{Tx}$ frequency response is compensated for by the receiver equalizer. The magenta (o) curve (right vertical axis) shows the PAPR of the RF signal into the modulator for different attenuation values at $20 \mathrm{GHz}$. Higher attenuation results in lower PAPR, which allows for the use of lower cost AWGs with smaller ENoB and RF amplifiers with less stringent requirements on linearity and smaller gain. The reduction of PAPR is, however, smaller than that found in simulation as we measure it after the AWG and DA, where low pass response of the Tx comes into play.

We also measured the $Q$ factor penalty associated with partial pre-emphasis for a 50 Gbaud 16QAM transmission after $80 \mathrm{~km}$ on SSMF at different OSNR values. We found that the Q factor penalty increases from 0 at $22 \mathrm{~dB}$ OSNR to 0.11 at $18 \mathrm{~dB}$ OSNR for an attenuation of $3 \mathrm{~dB}$ at $20 \mathrm{GHz}$. It is worthwhile to note that though the PAPR of the received signal increases due to chromatic dispersion (CD), the $Q$ factor penalty remains stable.

Figure 8 shows the BER for different levels of partial pre-emphasis in Back-to-Back (B2B) case for two different OSNR values. As mentioned earlier, the BER is similar after $80 \mathrm{~km}$ transmission. The BER threshold for the 400ZR FEC is chosen, as in [1], to be 0.0125 .

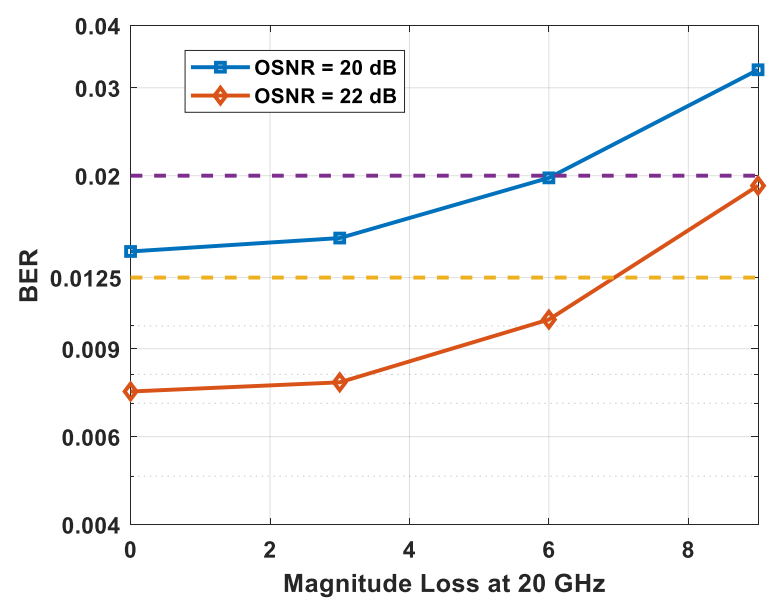

Figure 8. Bit-error rate (BER) versus loss at $20 \mathrm{GHz}$ for $50 \mathrm{Gbd}$ 16QAM signal.

4.1.2. Impact of Partial Pre-Compensation on Q-Factor in a Dual-Polarization Coherent System with PDL and PMD

Based on 400ZR specifications from [1], typical link impairments are expected to be handled by the adaptive filter at the Rx. In order to stress-test the Rx equalization capability so it performs further post-compensation while keeping the manageable Q penalty, as shown in Figure 9, we ran a dual-polarization experiment using the setup shown in Figure 7. Using emulators, polarization-dependent loss (PDL) of $3.5 \mathrm{~dB}$ and polarization-mode dispersion (PMD) of 28 ps were set. PC1 and PC2 are used to scramble the signal polarization, emulating state-of-polarization (SOP) variations at rotation rates of $1 \mathrm{rad} / \mathrm{ms}$ and $50 \mathrm{rad} / \mathrm{ms}$, respectively. Additionally, we included, as typically used in point-to-point DCI connections, a $75 \mathrm{GHz}$ C-band channel mux/demux (CMD) at the ingress and egress of the fiber model with a $3 \mathrm{~dB}$ net half bandwidth of $36 \mathrm{GHz}$. With a modulator attenuation at $20 \mathrm{GHz}$ equal to 3, 6, and $9 \mathrm{~dB}$, when comparing full versus partial pre-emphasis in presence of impairments, the Q-factor penalty is measured to be $0.25,0.5$, and $1.1 \mathrm{~dB}$, respectively. In comparison to the data presented in Figure 6, the results show that adding impairments does not introduce more than $0.1 \mathrm{~dB}$ of additional penalty. 


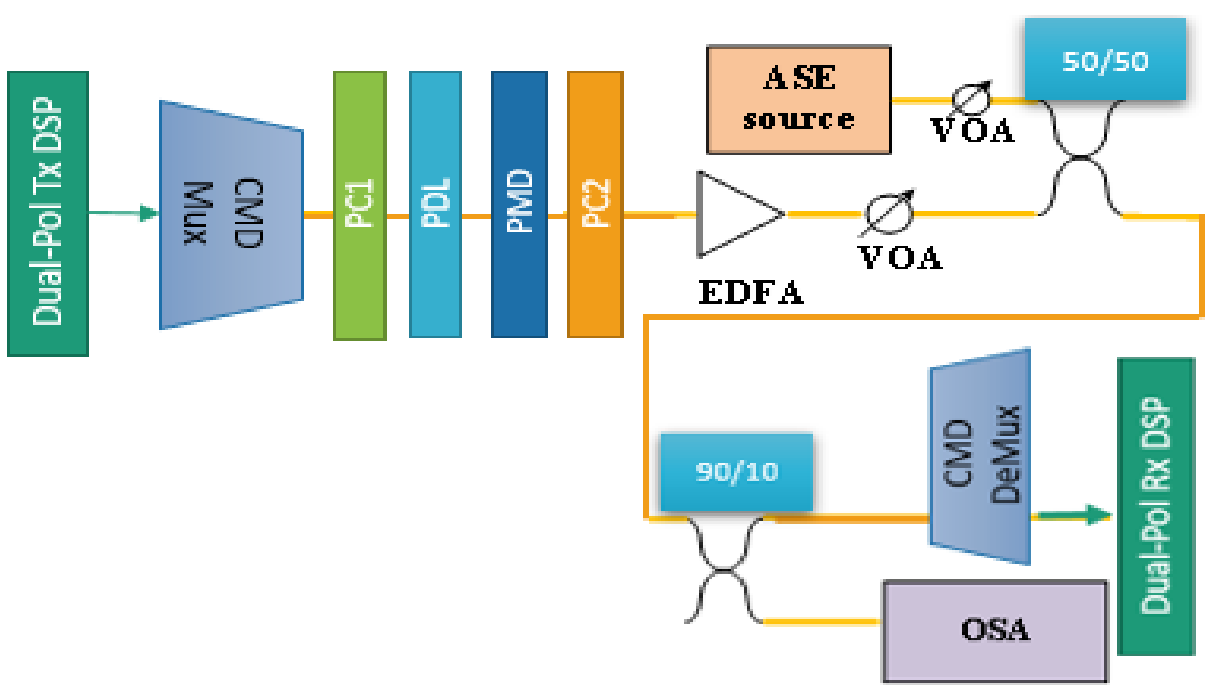

Figure 9. Dual-polarization stress-test experimental setup.

\section{Design Trade-Offs}

An important aspect of 400ZR system design is power consumption. Reducing the frequency response peaking of the pre-emphasis filter, i.e., relying more on the post-compensation of the channel ISI, increases the signal power out from DAC, thus lessening the required gain of the driver or EDFA.

We summarize that partial pre-emphasis provides the following advantages for coherent system design at the expense of manageable performance penalty: (i) this leads to less signal distortion from the nonlinearity of the RF DA and modulator due to reduced PAPR. It also helps mitigate the effect of fiber nonlinearity during transmission [19]. However, this has minimal performance impact on the targeted short-reach transmission, i.e., $100 \mathrm{~km}$ specified for 400ZR. (ii) It allows the transceiver to be more resilient to jitter induced by the PLL driving the DAC clocking. It is known that high-frequency components are more sensitive to jitter [20], therefore by performing less peaking (i.e., by reducing amplitudes of high-frequency components), smaller system SNR degradation is observed. As follows, the SNR degradation due to jitter $\left(T_{j i t t e r}\right)$ is calculated as a function of frequency components of the signal $\left(\right.$ Freq $\left._{\text {in }}\right)$

$$
S N R_{j i t t e r}[d B c]=-20 * \log \left(2 * \pi * \text { Freq }_{i n} * T_{j i t t e r}\right)
$$

(iii) It relaxes the "Adjacent channel isolation" per port specification for the channel Multiplex/DeMultiplex (CMD), since for point-to-point fixed grid systems (i.e., $75 \mathrm{GHz}$ ), partial pre-emphasis means that the inter channel interference will be mitigated due to less power leakage from adjacent channels. This also applies to gridless (or flexible-grid) coherent networks though not yet included in the 400ZR standard. (iv) Moreover, the fidelity of clock phase error estimators, such as Gardner [21] and Godard [22], that depends on the clean energy at the low and high end of the signal frequency response improves since partial pre-emphasis reduces the leaked noise into the edges of neighboring channels [23].

\section{Conclusions}

In this work, we evaluate the impact of implementing partial pre-emphasis for low-cost, low-power short-reach coherent systems. The numerical and experimental results indicate that partially pre-compensating for the Tx low-pass filtering leads to decreased signal PAPR, and this can help reduce the AWG ENoB requirement to a certain extent. In addition, the signal power increase from the AWG output suggests that RF DAs with lower gain can be employed to drive the modulator. It is also found that the performance penalty due to partial pre-emphasis is manageable for both single-pol and dual-pol coherent systems. 
Author Contributions: A.A., X.L., M.S.A., N.B.-H., and M.P. conceived the idea and performed simulations and measurements. C.D. and D.P. analyzed the data and helped write the paper.

Funding: This work was funded by Mitacs.

Conflicts of Interest: The authors declare no conflict of interest.

\section{References}

1. OIF. Implementation Agreement 400ZR, IA \# OIF-400ZR 0.10-Draft, oif2017.245.10.pdf. Available online: https://www.oiforum.com/technical-work/hot-topics/400zr-2/ (accessed on 10 December 2019).

2. Patel, D.; Ghosh, S.; Chagnon, M.; Samani, A.; Veerasubramanian, V.; Osman, M.; Plant, D.V. Design, analysis, and transmission system performance of a $41 \mathrm{GHz}$ silicon photonic modulator. Opt. Exp. 2015, 23, 14263-14287. [CrossRef] [PubMed]

3. Samani, A.; Chagnon, M.; Patel, D.; Veerasubramanian, V.; Ghosh, S.; Osman, M.; Zhong, Q.; Plant, D.V. A Low-Voltage 35-GHz Silicon Photonic Modulator-Enabled 112-Gb/s Transmission System. IEEE Photon. J. 2015, 7, 1-13. [CrossRef]

4. Dong, P.; Xie, C.; Chen, L.; Buhl, L.L.; Chen, Y.K. 112-Gb/s monolithic PDM-QPSK modulator in silicon. Opt. Exp. 2012, 20, B624-B629. [CrossRef] [PubMed]

5. Verbist, J.; Zhang, J.; Moeneclaey, B.; Soenen, W.; Van Weerdenburg, J.; Van Uden, R.; Okonkwo, C.; Bauwelinck, J.; Roelkens, G.; Yin, X. A 40-GBd QPSK/16-QAM integrated silicon coherent receiver. IEEE Photon. Technol. Lett. 2016, 28, 2070-2073. [CrossRef]

6. Shi, W.; Lin, J.; Sepehrian, H.; Zhalehpour, S.; Zhang, Z.; Rusch, L.A. Silicon photonic modulators for high-capacity coherent transmissions. In Proceedings of the Optical Fiber Communication Conference 2019, San Diego, CA, USA, 3-7 March 2019.

7. Doerr, C.; Chen, L.; Vermeulen, D.; Nielsen, T.; Azemati, S.; Stulz, S.; McBrien, G.; Xu, X.-M.; Mikkelsen, B.; Givehchi, M.; et al. Single-chip silicon photonics 100-Gb/s coherent transceiver. In Proceedings of the Optical Fiber Communication Conference 2014, San Francisco, CA, USA, 9-13 March 2014.

8. Petousi, D.; Zimmermann, L.; Gajda, A.; Kroh, M.; Voigt, K.; Winzer, G.; Tillack, B.; Petermann, K. Analysis of optical and electrical tradeoffs of traveling-wave depletion-type Si Mach-Zehnder modulators for high-speed operation. IEEE J. Sel. Topics Quantum Electron. 2014, 21, 199-206. [CrossRef]

9. Patel, D.; Parvizi, M.; Ben-Hamida, N.; Rolland, C.; Plant, D.V. Frequency response of dual-drive silicon photonic modulators with coupling between electrodes. Opt. Express 2018, 26, 8904-8915. [CrossRef]

10. Wettlin, T.; Ohlendorf, S.; Rahman, T.; Wei, J.; Calabrò, S.; Stojanovic, N.; Pachnicke, S. Beyond 200 Gb/s PAM4 transmission using Tomlinson-Harashima precoding. In Proceedings of the 2019 European Conference on Optical Communication (ECOC), Dublin, Ireland, 22-26 September 2019.

11. Hu, Q.; Schuh, K.; Chagnon, M.; Buchali, F.; Bülow, H. 84 GBd Faster-Than-Nyquist PAM-4 Transmission Using Only Linear Equalizer at Receiver. In Proceedings of the 2019 Optical Fiber Communications Conference and Exhibition (OFC), San Diego, CA, USA, 3-7 March 2019.

12. Wei, J.; Zhang, L.; Prodaniuc, C.; Stojanović, N.; Xie, C. Linear Pre-Equalization Techniques for Short Reach Single Lambda $225 \mathrm{~Gb} / \mathrm{s}$ PAM IMDD Systems. In Proceedings of the 2018 European Conference on Optical Communication (ECOC), Rome, Italy, 23-27 September 2018; pp. 1-3.

13. Wei, J.; Stojanovic, N.; Zhang, L.; Calabrò, S.; Rahman, T.; Xie, C.; Charlet, G. Experimental comparison of modulation formats for 200 G/入 IMDD data center networks. In Proceedings of the 2019 European Conference on Optical Communication (ECOC), Dublin, Ireland, 22-26 September 2019.

14. Napoli, A.; Mezghanni, M.M.; Rafique, D.; Sleiffer, V.A.; Rahman, T.; Spinnler, B.; Calabrò, S.; Bohn, M. Novel DAC digital pre-emphasis algorithm for next generation flexible optical transponders. In Proceedings of the Optical Fiber Communication Conference, Los Angeles, CA, USA, 22-26 March 2015.

15. Rafique, D.; Napoli, A.; Calabro, S.; Spinnler, B. Digital preemphasis in optical communication systems: On the DAC requirements for terabit transmission applications. J. Lightw. Technol. 2014, 32, 3247-3256. [CrossRef]

16. Khanna, G.; Spinnler, B.; Calabrò, S.; De Man, E.; Hanik, N. A Robust Adaptive Pre-Distortion Method for Optical Communication Transmitter. IEEE Photon. Technol. Lett. 2015, 28, 752-755. [CrossRef] 
17. Rafique, D. Interplay of Pulse Shaping and Pre-Emphasis for High Symbol Rate Coherent Transmission Systems. In Proceedings of the 2018 20th International Conference on Transparent Optical Networks (ICTON), Bucharest, Romania, 1-5 July 2018.

18. Napoli, A.; Mezghanni, M.M.; Rahman, T.; Rafique, D.; Palmer, R.; Spinnler, B.; Calabrò, S.; Castro, C.; Kuschnerov, M.; Bohn, M. Digital compensation of bandwidth limitations for high-speed DAC and ADCs. J. Lightw. Technol. 2016, 34, 3053-3064. [CrossRef]

19. Park, M.; Jun, H.; Cho, J.; Cho, N.; Hong, D.; Kang, C. PAPR Reduction in OFDM Transmission Using Hadamard Transform. In Proceedings of the 2000 IEEE International Conference on Communications. ICC 2000. Global Convergence Through Communications. Conference Record, New Orleans, LA, USA, 18-22 June 2000.

20. Azeredo-Leme, C. Clock Jitter Effects on Sampling: A Tutorial. IEEE Circuits Syst. Mag. 2011, 11, $26-37$. [CrossRef]

21. Gardner, F. A BPSK/QPSK timing-error detector for sampled receivers. IEEE Trans. Commun. 1986, 34, 423-429. [CrossRef]

22. Godard, D. Passband Timing Recovery in an All-Digital Modem Receiver. IEEE Trans. Commun. 1978, 26, 517-523. [CrossRef]

23. Abdo, A.; Aouini, S.; Riaz, B.; Ben-Hamida, N.; D'Amours, C. Adaptive Coherent Receiver Settings for Optimum Channel Spacing in Gridless Optical Networks. Future Internet 2019, 11, 206. [CrossRef]

(C) 2019 by the authors. Licensee MDPI, Basel, Switzerland. This article is an open access article distributed under the terms and conditions of the Creative Commons Attribution (CC BY) license (http://creativecommons.org/licenses/by/4.0/). 